\title{
Uncinaria (Megadeirides) olseni n. sp., Nématode à caractères archaïques parasite d'un Tupaia à Bornéo
}

\author{
par A.-G. CHABAUD et Marie-Claude DURETTE-DESSET \\ Laboratoire de Zoologie (Vers) associé au C.N.R.S., \\ Muséum national d'Histoire naturelle, 43, rue Cuvier, F 75231 Paris Cedex 05 \\ et Division of Medical Ecology, Institute for Medical Research, \\ Jalang-Pahang, Kuala-Lumpur, Malaysia.
}

Résumé.

Description d'Uncinaria (Megadeirides) olseni n. sp. parasite de Tupaia glis à Borneo. L'espèce est rapprochée d'U. bauchoti, parasite des Tenrecs malgaches (sous-genre Megadeirides). Ces deux espèces paraissent faire la transition avec la sous-famille plus primitive des Globocephalinae. Le genre Uncinaria, lui-même, est interprété comme un groupe archaïque qui peut être placé à la base des Uncinariinae-Ancylostomatinae.

\section{Summary.}

Uncinaria (Megadeirides) olseni n. sp., Nematode with archaical morphological characters, parasite of a Tupaia from Borneo.

The species is closely related to $U$. bauchoti parasite of malagasian Tenrecoidea (sub-genus Megadeirides). The two species may be considered as a link with the primitive sub-family Globocephalinae. The genus Uncinaria itself is considered as an archaic group, which could be at the origin of Uncinariinae-Ancylostomatinae.

En janvier 1974, grâce à l'obligeance de M. G. Stanley de Silva, notre collègue et ami Jean-Claude Quentin a pu récolter des Helminthes parasites de Vertébrés à Sepilok, région de Sandakan, Sabah (Bornéo).

Dans l'intestin grêle de l'un des Tupaia glis examinés, il a trouvé un mâle, une femelle et un fragment antérieur du corps d'un Ancylostomatidae, dont il a bien voulu nous confier l'étude. 


\section{Description}

Nématodes dont la bouche, dirigée dorsalement, est sub-ovalaire. Elle est entourée par la terminaison de six pédoncules sensoriels. Chaque pédoncule sub-médian semble porter 3 papilles, les pédoncules latéraux portent l'amphide et, semble-t-il, une seule papille. La capsule buccale occupe tout l'espace céphalique. Son bord antérieur constitue 4 lames faiblement saillantes sous l'ouverture buccale. La forme générale de la bouche est celle des autres Uncinaria, mais le renforcement chitinoïde des lames est très faible et n'est pas visible en vue latérale comme chez les autres espèces du genre. Ses dimensions chez le $\hat{\delta}$ sont de $120 \mu$ du côté ventral, $105 \mu$ du côté dorsal; le diamètre externe est de $80 \mu$ en avant et de $50 \mu$ en arrière. Incluses dans la face ventrale de la capsule se trouvent deux petites dents hautes de $20 \mu$ (fig. 1, B), qui ne déterminent que deux faibles saillies dans la cavité buccale.

MÂle: Corps long de $3,8 \mathrm{~mm}$, large de $120 \mu$ dans sa partie moyenne. Anneau nerveux, pore excréteur et deirides situés respectivement à $325 \mu, 350 \mu$ et $350 \mu$ de l'apex. Esophage long de $510 \mu$.

Bourse caudale figurée en $1, G$ et $H$. Elle présente la particularité d'avoir des côtes 4 très courtes dont l'extrémité, dirigée postérieurement, croise les côtes 5. Papilles prébursales bien visibles. Spicules longs de $375 \mu$, fins, ailés, à extrémité postérieure légèrement tordue. Gubernaculum haut de $75 \mu$. Cône génital non étudié (un seul mâle disponible).

Femelle : Corps long de 3,9 mm, large de $125 \mu$ dans sa partie moyenne. Anneau nerveux, pore excréteur et deirides situés respectivement à $300 \mu, 325 \mu$ et $330 \mu$ de l'apex. Esophage long de $540 \mu$. Ovéjecteur long de $330 \mu$, figuré en $1, F$. Vulve située à 1,3 mm de l'extrémité postérieure. Utérus embryonnaire, sans œufs formés. Queue longue de $135 \mu$ avec une petite pointe terminale longue de $13 \mu$.

\section{Discussion}

Notre espèce se distingue aisément des autres espèces déjà connues dans le genre Uncinaria Froelich, 1789 par la côte 4 courte et recourbée vers l'arrière à tel point qu'elle croise la 5 . Il s'agit donc d'une espèce nouvelle que nous nommons Uncinaria (Megadeirides) olseni $\mathrm{n}$. sp.

Olsen, 1968 a donné la liste et la clé des douze espèces décrites dans le genre. Il faut y ajouter Uncinaria miyazakiensis (Nagayosi, 1955), Noda et Yamada, 1964 ; il est possible que cette espèce corresponde en réalité à $U$. stenocephala mais nous n'avons pu avoir connaissance de l'article original.

Le cas de Necator urichi Cameron, 1936, est plus difficile. L'espèce n'ayant qu'une seule paire de languettes, dans le fond de la capsule buccale, a été reclassée 

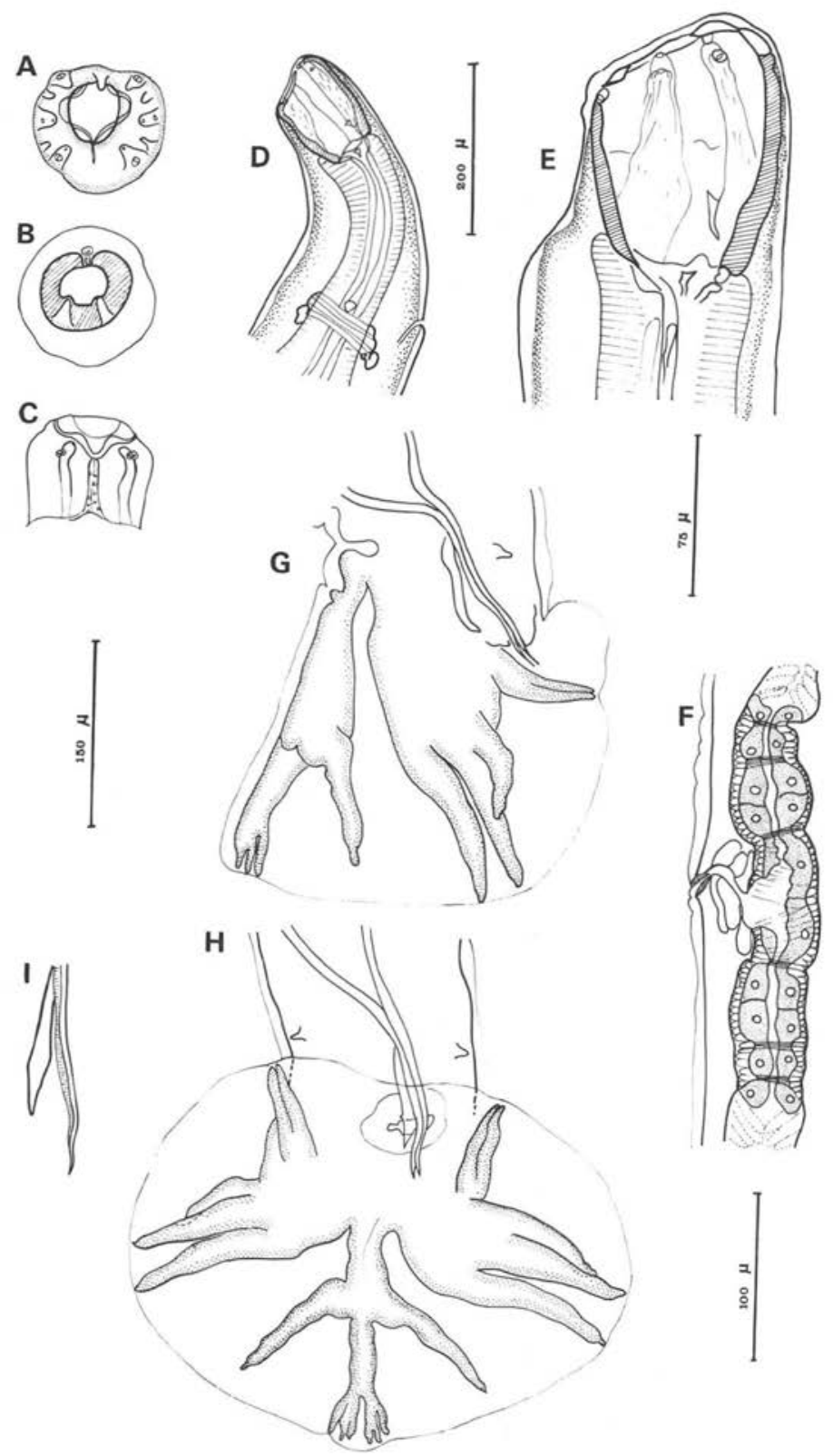

FIG. 1. - Uncinaria olseni n. sp. A : $\uparrow$, tête en vue apico-dorsale ; B : $\uparrow$, coupe de la capsule buccale au niveau des dents latéro-ventrales; $\mathrm{C}$ : $q$, tête en vue dorsale ; D : $\delta$, extrémité antérieure, vue latérale droite; E : ठ', tête, vue latérale droite; F : + , région de

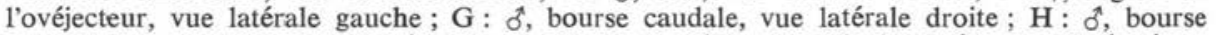
caudale, vue ventrale; I: $\delta$, gubernaculum et pointe du spicule droit non disséqués.

A, B, C, F, éch. : $150 \mu$; D, éch. : $200 \mu ; \mathrm{E}, \mathrm{I}$, éch. : $75 \mu ; \mathrm{G}, \mathrm{H}$, éch. : $100 \mu$. 
dans le genre Uncinaria par Chabaud, Bain et Houin, 1967. Cependant, la description du mâle donnée par Teixeira de Freitas, 1951, montre que chaque languette est bidentée, que le cône du conduit de la glande osophagienne dorsale se détache de la capsule buccale et surtout que la côte dorsale, profondément divisée, est de type Necator et non Uncinaria. Il s'agit donc, semble-t-il, d'une forme de transition entre Uncinaria et Necator et nous pensons aujourd'hui qu'il n'est pas souhaitable, en s'appuyant simplement sur l'hypertrophie remarquable des deirides, de rapprocher cette espèce d'U. bauchoti Chabaud et Tcheprakoff, 1964.

En revanche, $U$. olseni semble présenter de véritables affinités avec $U$. bauchoti espèce type du sous-genre Megadeirides Chabaud, Bain et Houin, 1967.

Ces deux espèces constituent un groupe faisant transition entre Globocephalinae et Uncinariinae. En effet, les lames tranchantes buccales, qui caractérisent les Uncinariinae, sont visibles à l'intérieur de la bouche mais sont peu chitinoïdes et ne sont pas perceptibles en vue latérale. En outre, la bourse caudale ressemble à celle de Globocephalus car elle est arrondie avec un lobe dorsal peu marqué et une côte 4 très courte. Dans les deux cas, les hôtes sont des animaux reliques: Tenrec à Madagascar, Tupaia à Bornéo. Nous amendons donc la définition du sous-genre Megadeirides qui doit se différencier d'Uncinaria sensu stricto non sur l'hypertrophie des deirides mais sur le faible développement des lames coupantes de la capsule buccale et sur la faible longeur de la côte 4 .

En conclusion, à défaut d'un sous-genre particulier qui permettrait de séparer urichi des formes africaines, nous préférons replacer l'espèce dans le genre Necator, où elle a été primitivement décrite, et nous plaçons $U$. olseni à côté d'U. bauchoti comme deuxième espèce du sous-genre Megadeirides.

Par le sous-genre Megadeirides qui fait transition avec les Globocephalinae, le genre Uncinaria paraît être ainsi la forme la plus primitive des Uncinariinae et des Ancylostomatinae. Par ailleurs la liste de ses hôtes semble bien indiquer une grande ancienneté : sur les 14 espèces, nous trouvons les 2 Megadeirides parasites d'animaux reliques, 2 espèces chez les Pinnipèdes, 7 espèces chez les Mustelidés, Ailuridés, Procyonidés et Ursidés qui sont précisément les 4 familles les plus archaïques de Carnivores (cf. Thenius, 1972, p. 205); 2 espèces seulement (ou 3 espèces si miyazakiensis est valide) sont adaptées aux Felidés et aux Canidés.

On remarquera également qu'il s'agit d'un genre cosmopolite alors que les autres genres d'Uncinariinae ou d'Ancyclostomatinae sont caractéristiques d'une région géographique déterminée (voir par ex. Biocca et Le Roux, 1958).

Tous les éléments concordent donc pour permettre de placer le genre Uncinaria, et en particulier le sous-genre Megadeirides à la base des Uncinariinae-Ancylostomatinae et de supposer que sa phase d'expansion remonte au début de l'Eocène. 


\section{Bibliographie}

Biocca (E.) et Le Roux (P.-L.), 1958. - Subdivision del generae Ancylostoma (Dubini, 1843 ) in quattro Sottogeneri, Atti. Accad. Nazion. Lincei. Rond Classe. Sc. Fisiche, Matematiche. Nat. Rome, Sér. 8, 23, 470-477.

Cameron (T. W. M.), 1936. - Studies on the endoparasitic fauna of Trinidad. III. Some parasites of Trinidad Carnivores. Can. Jour. Res., 14, 25-38.

Chabaud (A.-G.), Bain (O.) et Houin (R.), 1966. - Nématodes de Potamochères malgaches. Ann. Parasit. hum. comp., 41, 599-606.

Chabaud (A.-G.), Brygoo (E.-R.) et Tcheprakoff (R.), 1964. - Nématodes parasites d'Insectivores malgaches. Bull. Mus. Hist. Nat., Paris, $2^{\circ}$ sér., 36, 245-261.

Nagayosi (Y.), 1955. - Two new species of hookworms from Nyctereutes procyonides, Ancylostoma kusimaense n. sp. and Necator miyazakiensis n. sp. Tokyo IjiShinsni, 72, 349-353 (ouvrage non consulté).

Noda (R.) et Yamada (H.), 1964. - On two species of nematodes, Necator gorillae sp. nov. (Ancylostomidae) and Chitwoodspirura wehri Chabaud and Rousselot, 1956 (Spiruridae), from a gorilla. Bull. Univ. Osaka Pref, Sér. B, 15, 175-180.

OLsen (O.W.), 1968. - Uncinaria rauschi (Strongyloidea: Nematoda) a new species of hookworms from Alaskan bears. Canad. J. Zool., 46, 1113-1117.

Texeira de Freitas (J. F.), 1951. - Alguns « Strongyloidea» parasitas de « Procyon cancrivorus» (Cuv.) (Nematoda). Rev. Brasil. Biol., 11, 189-202.

Thenius (E.), 1972. - Grundzüge der Verbreitungsgeschichte der Saügetiere. Gustav Fischer Verlag, Stuttgart, 345 p. 